\title{
Labyrinthe
}

28 | 2007 (3)

Des Juifs contre l'émancipation

\section{Le tsar, l'empereur et le roi des rois : remarques sur les géographies de l'existence}

\section{Pierre Savy}

\section{(2) OpenEdition \\ 1 Journals}

Édition électronique

URL : http://journals.openedition.org/labyrinthe/2883

DOI : $10.4000 /$ labyrinthe.2883

ISSN : 1950-6031

Éditeur

Hermann

Édition imprimée

Date de publication : 21 décembre 2007

Pagination : 63-75

ISBN : 978-2-9526131-5-6

Référence électronique

Pierre Savy, «Le tsar, l'empereur et le roi des rois : remarques sur les géographies de l'existence »,

Labyrinthe [En ligne], 28 | 2007 (3), mis en ligne le 01 octobre 2009, consulté le 30 avril 2019. URL

http://journals.openedition.org/labyrinthe/2883 ; DOI : 10.4000/labyrinthe.2883

Propriété intellectuelle 


\title{
LE TSAR, L'EMPEREUR ET LE ROI DES ROIS : remarques sur les géographies de l'existence
}

\author{
Pierre SAVY \\ savy_pierre@yahoo.fr
}

Hantés par la fameuse phrase de Levinas, selon laquelle «s'interroger sur l'identité juive, c'est déjà l'avoir perdue ${ }^{1} »$, certains penseurs de la fin du $\mathrm{XX}^{\mathrm{e}}$ siècle et du début du suivant évoquent un temps béni où nul ne se demandait qui était juif et qui ne l'était pas, si on l'était vraiment, ce que c'était donc que de l'être; un âge d'or perdu - comme tout âge d'or - où les Juifs ne bénéficiaient certes pas des mêmes droits que les autres habitants du pays, et étaient contraints de vivre dans un ghetto ou, du moins, dans l'inégalité politique, mais où, précisément, cette contrainte protégeait leur survie en tant que Juifs.

La séparation est équivoque: les portes du ghetto enferment les Juifs mais, dans le même temps, elles les isolent et les protègent des violences. Elles les protégeraient aussi des bontés dont on voudrait les faire bénéficier, qui seraient des «cadeaux empoisonnés»: «Timeo gentiles et dona ferentes.» Entre les lois de séparation formulées par les chrétiens et celles formulées par les Juifs, on observe une similitude structurelle. À quoi l'on opposera que la domination chrétienne donne à la législation antijudaïque une signification toute différente de celle qu'ont les lois de séparation juives: et pourtant, d'aucuns s'imaginent des bienfaits du ghetto.

Il serait difficile de tracer la généalogie exacte de cette nostalgie d'une période pourtant le plus souvent présentée comme nuisible: au $\mathrm{XIX}^{\mathrm{e}}$ siècle ou avant-guerre, dans les milieux sionistes ou religieux, l'émancipation fut critiquée par ses bénéficiaires supposés, dont certains formulèrent même cette idée d'un «retour au ghetto ». Contentonsnous ici de quelques bribes: le «'Hatam Sofer» (1762-1839) se fit

1. Emmanuel Levinas, Difficile Liberté. Essais sur le judaïsme, Paris, A. Michel, 1963, rééd. Paris, Librairie générale française, 1984, p. 78. 
depuis Presbourg le champion du maintien intégral de la tradition et du refus de l'émancipation, avec sa fameuse formule «les nouveautés sont interdites par la Torah». Plus tard, les «bontés» prodiguées aux Juifs suscitèrent diverses inquiétudes: à propos de la figure d'Ésaü, classiquement comprise depuis le Maharal comme l'antonomase de l'Occident, Rabbi Yossef Baer Soloveitchik de Brisk (1820-1892) affirme que Jacob redoute ses moments de bienveillance autant que les épisodes où il se montre agressif, et de même Rabbi Yossef 'Hayim de Bagdad (1832-1909) craint que les bontés d'Ésaü ne passent par l'abandon de la spécificité juive ${ }^{1}$. À la fin du XIX ${ }^{\mathrm{e}}$ siècle, le sioniste Max Nordau appelle à un paradoxal retour au ghetto. De nouveau, juste avant guerre, des intellectuels juifs installés à Paris, devant ce qu'ils perçoivent comme un échec patent de l'émancipation (en raison de l'assimilation et de l'antisémitisme), préconisent le même retour².

Le programme choque ceux qui sont attachés à la liberté donnée par les démocraties ${ }^{3}$. Mais penchons-nous ici non pas sur ces étapes intermédiaires, ni a fortiori sur celles de la reconstruction fantasmatique, mais sur la matrice même de ces reconstructions : le moment de la réaction face au phénomène d'émancipation, quand s'ouvrirent les ghettos, réels ou symboliques, ou du moins quand leur ouverture devint envisageable. Si l'on cherche les premières expressions d'un refus de l'émancipation, on trouve, dans une maigre récolte, quelques réticences formulées en Europe occidentale, mais, dans l'ensemble, les choses y demeurèrent bien modérées; tout au plus observe-t-on, à propos du phénomène d'assimilation, «l'embarras des conservateurs ${ }^{4} »$. On trouve au contraire un net refus, promis à rencontrer beaucoup d'écho, dans les textes 'hassidiques ${ }^{5}$ produits en Europe centrale et orientale, où la

1. Voir notamment Binyamin Tagger, "'Sauve-moi des mains de mon frère, des mains de "Essaw"...», Kountrass, 16, 1989, «Les Juifs et la Révolution», p. 28-30.

2. Voir Simon Dubnov, Lettres sur le judaïsme ancien et nouveau, éd. Renée Poznanski, Paris, Le Cerf, 1989, respectivement p. 321 et p. 37.

3. La liste est longue des ouvrages exprimant pareille indignation; voir par exemple Pierre VidalNaquet, L'histoire est mon combat : entretiens avec Dominique Bourel et Hélène Monsacré, Paris, A. Michel, 2006, notamment p. 112-113.

4. Pour reprendre le titre du chapitre IX de Jacob Katz, Out of the Ghetto, The Social Background of Jewish Emancipation. Cambridge, Harvard University Press, 1973, traduction française Hors du ghetto. L'émancipation des Juifs en Europe. 1770-1870, Paris, Hachette, 1984, p. 155-174, sur les traditionalistes qui, navrés de voir des Juifs s'éloigner, se gardèrent toutefois de condamner l'émancipation.

5. Nous voulons parler des textes produits par les 'hassidim, Juifs orthodoxes participant au 'hassidisme, vaste mouvement de piété et de ferveur apparu au XVIII siècle en Europe orientale. 
réalité dominante n'était pas le ghetto, mais le shtetl. Rappelons que le premier est à strictement parler un fait italien, complexe et spécifique; il désigne un quartier où les Juifs sont contraints de demeurer et où n'habitent pas de non-Juifs. Le shtetl, dont la figure souvent mythologisée et présentée comme an-historique ne doit pas faire négliger la variété des statuts et des conditions déjà au début du XIX ${ }^{e}$ siècle, renvoie simplement à une agglomération d'Europe centrale et orientale où résident de nombreux Juifs, sans qu'ils soient nécessairement obligés de le faire ${ }^{1}$.

\section{Alexandre $\mathbf{I}^{\text {er }}$ ou Napoléon?}

En 1812, ont lieu divers événements certes ponctuels, mais précoces et fort significatifs dans l'histoire de ces communautés. Ces faits illustrent l'opposition entre deux conceptions antagonistes, et vont même contribuer à structurer cette opposition dans la suite de l'histoire du judaïsme. C'est par ce cas historique que notre réflexion peut commencer.

La nécessité du respect pour le roi en place figure dans la loi juive ${ }^{2}$; de surcroît, les 'hassidim voient dans un roi une «métaphore» (un «mashal» en hébreu) de la figure divine. Selon l'enseignement 'hassidique, un objet similaire et «analogique» aide à la compréhension, d'autant plus quand l'objet de la connaissance est précisément ce qui échappe à la connaissance - Dieu. Ne parle-t-on pas souvent de lui comme du «Roi des rois»? Voilà qui met en contexte l'attitude qu'eurent les 'hassidim 'habad lorsque les armées napoléoniennes menacèrent de l'emporter en Europe orientale. Ils ne furent du reste pas les seuls à débattre du rôle de Napoléon, ni même les seuls à souhaiter sa défaite.

1. Même s'il arrivait qu'il y eût contrainte - ainsi avec les fameuses «Lois de mai» (1882), voulues par le tsar Alexandre III. Voir une bonne présentation de la société juive traditionnelle dans Jacob Katz, De la tradition à la crise. La société juive à la fin du Moyen Âge (Jérusalem, Mosad Byalik, 1958), traduction française Paris, Cerf, 2000. Sur l'histoire du 'hassidisme à ses débuts, Jean Baumgarten, La Naissance du 'hassidisme. Mystique, rituel et société, XVIII'-XIXe siècle, Paris, A. Michel, 2006.

2. Elle a donné lieu à une étude récente, Pierre Birnbaum, Prier pour l'État. Les Juifs, l'alliance royale et la démocratie, Paris, Calmann-Lévy, 2005; voir en particulier «Le modèle de l'alliance royale», p. 7-34. 
Parmi les protagonistes du débat, un homme occupe une place centrale: Rabbi Chnéour Zalman (1745-1812), souvent appelé l'Alter Rebbe ou le Baal hatanya, du nom de son principal ouvrage, le Tanya. Ce disciple d'un disciple du Baal Chem Tov (Rabbi Israël ben Eliezer, 16981760 , le fondateur du 'hassidisme) fonda la branche 'habad du 'hassidisme (branche qui s'appellerait plus tard Loubavitch ${ }^{1}$ ). À la fin de l'été 1812, Zalman s'est opposé à l'avancée de Napoléon Bonaparte en Russie, tandis que d'autres personnalités importantes du judaïsme, y compris parmi les 'hassidim, apportaient leur soutien aux armées françaises, dans l'espoir que le succès de la campagne de Russie améliorerait le sort des Juifs et mettrait un terme à l'alliance de l'État avec la religion chrétienne. Au contraire, Zalman prit le parti du tsar Alexandre ${ }^{\mathrm{er}}$. La principale source est une célèbre lettre qu'il adressa à son disciple, Rabbi Moché Meislich de Vilna:

Le premier jour de Rosh hashana [le nouvel an juif - le 7 septembre 1812], avant musaf [prière additionnelle], il m'a été révélé que si Bonaparte était victorieux, la prospérité matérielle d'Israël augmenterait, mais le cœur d'Israël serait séparé et exilé de son Père dans les cieux. Mais si notre maître Alexandre était victorieux, même si la pauvreté s'accroîtrait pour Israël et que sa gloire serait amoindrie, les cœurs d'Israël resteraient liés et attachés solidement à son Père dans les cieux ${ }^{2}$.

\footnotetext{
1. Son mouvement ne s'est installé que plus tard dans la ville de Loubavitch: il est donc impropre de l'appeler déjà à l'époque de Chnéour Zalman de son autre nom actuel de mouvement Loubavitch.

2. Chnéour Zalman, Igrot kodesh, n 64 (Igrot Baal hatanya, Jérusalem, Hamassorah, 1953, p. 238 [non consulté]). Sur ces aspects de l'histoire du 'hassidisme, voir notamment Jean Baumgarten, Récits hagiographiques juifs, Paris, Le Cerf, 2001, p. 479-491 et Jean Baumgarten, La Naissance..., op. cit., p. 386-410 et p. 610; Martin Buber, Gog et Magog. Chronique de l'époque napoléonienne, éd. fr. Paris, 1958; Simon Dubnov, History of the Jews, 4, New York, Londres et South Brunswick, T. Yoseloff, 1971, p. 733, et Simon Dubnov, History of the Jews in Russia and Poland from the Earliest Times Until the Present Day, 1, Philadelphie, The Jewish Publication Society of America, 1946, rééd. New York, Ktav Pub. House, 1975, p. 356-357; Henri Kahn, «Les Rabbis 'hassidiques et Napoléon: pour ou contre?», Kountrass, 9, 1988, «Napoléon et les Juifs», p. 30-35; Barukh Mevorakh, Napoleon utekufato, Jérusalem, Mosad Byalik, 1968, p. 181-183, qui publie la lettre; Shmuel Zanvl Pipe, «Napolyon in Yidishn folklor», dans Elias Tcherikower, Yidn in Frankraykh, 1, New York, Yidisher Visnshaftlekher Institut, 1941, p. 153-189; et Simon Schwarzfuchs, Napoleon, the Jews and the Sanhedrin, Londres, Boston et Henley, Routledge \& Kegan Paul, 1979, p. 176. Sur Chnéour, l'opuscule hagiographique dont s'inspirent les compilateurs est Shivhei harav, composé par Michael Levi Frumkin (1864); sur sa vie et sa spiritualité, voir Nissan Mindel, Rabbi Schneur Zalman of Liadi, New York, Kehot Publication Society, 1969, 2 vol. (surtout 1, p. 250-253 pour ce qui nous intéresse) et Roman A. Foxbrunner, Habad. The Hasidism of R. Shneur Zalman of Lyady, Tuscaloosa et Londres, University of Alabama Press, 1992.
} 
L'interprétation de cette lettre est malaisée, d'autant que, comme chacun sait, les armées françaises ne remportèrent pas la guerre: la situation que Zalman redoutait demeure inconnue. On peut distinguer trois interprétations possibles. La lecture la plus évidente consiste à comprendre que le mieux-être que permettrait la victoire française ne vaudrait pas l'éloignement de la religion qu'elle occasionnerait. Ce que l'on gagnerait sur le plan matériel ne vaudrait rien au regard de la perte sur le plan spirituel; peut-être le corps exulterait-il, mais l'âme sombrerait assurément. Selon cette première lecture, que nous croyons la plus fidèle au discours de Zalman, si l'on fait la «pesée» des éléments positifs et des éléments négatifs, on juge préférable la victoire du tsar. On peut aller plus loin et dire, et c'est la deuxième lecture, que du mal même on pourrait, dans une approche ascétique voire doloriste, tirer un bien, ce qui entraînerait que le maintien de la domination du tsar serait doublement positive: en raison, bien sûr, de la proximité plus grande avec la Torah, mais aussi par ce qu' elle comporte de nuisible sur le plan de la matérialité. Enfin, dans une perspective nettement critique et contraire aux deux précédentes, d'aucuns ont soutenu qu'il n'y avait là rien d'un ascétisme, d'ailleurs peu conforme à la spiritualité de l'Alter Rebbe, et qu'il fallait pour comprendre ce texte lui chercher des motivations politiques - l'intérêt du mouvement dans la domination russe maintenue - plutôt que spirituelles ${ }^{1}$.

Quoi qu'il en soit, le projet révolutionnaire était compris comme porteur non seulement de l'émancipation politique, mais aussi du désir d'émanciper tous les citoyens, juifs y compris, de leur attachement à leur «foi», pensée comme une aliénation: émanciper les Juifs de leur propre soumission à la religion n'est pas l'objectif d'autres traditions émancipatrices (celle des États-Unis d'Amérique, en particulier), dont le mouvement Loubavitch, aujourd'hui implanté à Brooklyn et fortement lié aux États-Unis d'Amérique, fait l'éloge ${ }^{2}$, mais tel semblait

1. C'est le propos du travail intéressant mais confus de Hillel Levine, «"Should Napoleon be victorious...": Politics and Spirituality in Early Modern Jewish Messianism», Jerusalem Studies in Jewish Thought, 16-17, 2001, p. 65-83, qui élève des doutes sur l'authenticité du document et propose de combiner deuxième et troisième interprétations.

2. Voir la note 9 de Yanki Tauber, «The Giver» (texte disponible sur internet), qui exprime de façon hyperbolique l'amour aujourd'hui porté aux États-Unis d'Amérique par le mouvement Loubavitch. 
bien le projet et même la spécificité de l'émancipation française, du moins dans sa forme la plus dure ${ }^{1}$.

Zalman faisait preuve de constance: en 1798, n'avait-il pas fait une prière pour qu'échouât la Révolution française ${ }^{2}$ ? Cela nous engage à supposer que la situation qu'il craignait était, mutatis mutandis, celle que connut le judaïsme français. Il n'avait pas été le premier: déjà Rabbi Lévi Yitz'hak de Berditchev (1740-1810) s'était engagé politiquement contre Napoléon ${ }^{3}$. Notons que l'hostilité à Napoléon, que l'on rattache le plus souvent à des considérations exclusivement spirituelles, tenait aussi à une prise en considération d'un risque matériel: dans une lettre qu'il adresse à Rabbi Meislich encore, Dov Baer, le fils de l'Alter Rebbe, exprime de nouveau de l'hostilité envers Napoléon, pour des raisons «religieuses» mais aussi en raison de l'affirmation du père de l'auteur selon laquelle, si Napoléon

ne poussait l'ambition jusqu'à la démence en tentant de pénétrer jusqu'en Russie, il se pouvait qu'il renforce sa position en Pologne, ce qui serait une catastrophe pour les Juifs polonais dont aucun ne subsisterait dans son judaïsme, ni ne resterait maître de ses biens ${ }^{4}$.

Zalman se livra à des interventions de type théurgique contre Napoléon; il s'engagea aussi sur le plan concret, en utilisant Moché Meislich comme espion - cet homme, interprète du haut commandement français, dévoilait les plans français aux généraux du tsar ${ }^{5}$.

Rares étaient ceux qui défendaient l'empereur, mais certains dirigeants, y compris 'hassidiques, en Pologne surtout, espéraient sa victoire,

1. Voir récemment Pierre Birnbaum, L'Aigle et la Synagogue. Napoléon, les Juifs et l'État, Paris, Fayard, 2007 ; pour un aperçu de l'émancipation à partir des sources, Les Juifs de France. De l'émancipation à l'intégration (1787-1812). Documents, bibliographie et annotations, éd. Richard Ayoun, Paris et Montréal, L'Harmattan, 1997; voir enfin deux essais à la tonalité proche, Monique-Lise Cohen, Les Juifs ont-ils du cœur? Discours révolutionnaire et antisémitisme, Toulouse, Vent Terral, 1992, et Henri Meschonnic, L’Utopie du Juif, Paris, Desclée de Brouwer, 2001.

2. Roland Goetschel, «L'hostilité du monde hassidique à la Révolution française», dans Mireille Hadas-Lebel et Évelyne Oliel-Grausz dir., Les Juifs et la Révolution française. Histoire et mentalités, colloque de Paris, 16-18 mai 1989, actes publiés à Louvain et Paris, E. Peeters, 1992, p. 267-283, p. 278. C'était à l'occasion du commentaire de la parashat «Haazinu».

3. Roland Goetschel, «L'hostilité...», art. cit., p. 273.

4. «Le Rabbi de Ladi et Napoléon», Kountrass, 9, 1988, «Napoléon et les Juifs», p. 40-44, p. 40. (C'est nous qui soulignons.)

5. Voir Henri Kahn, «L'espion du Rabbi chez l'Empereur...», Kountrass, 9, 1988, « Napoléon et les Juifs », p. 36-39. 
notamment au motif qu'ils voyaient dans une victoire française la préparation de la venue du Messie et la rédemption finale ${ }^{1}$. Nombreux furent parmi les frankistes (les disciples du faux messie Jacob Frank, 1726-1791) ceux qui allèrent jusqu'à voir en Napoléon le Messie. Quant aux mitnagdim, les opposants au 'hassidisme, il semble que, pour des raisons mal éclaircies (du simple fait de leur localisation, une moindre implication dans ces controverses? une moindre propension à des lectures mystiques? une structuration rendant moins nécessaire le maintien de micro-communautés autonomes ${ }^{2}$ ?), ils furent dans leur ensemble plus enclins à considérer favorablement l'hypothèse d'une victoire française. Parmi les 'hassidim, Rabbi Mena'hem Mendel de Rimanov, l'un des partisans de Napoléon, s'opposa vivement à son disciple, Naphtali Zevi (1760-1827). Ce dernier, proche des positions de Zalman, exprima la crainte que son succès eût des effets négatifs sur les Juifs de Pologne, comme le service militaire, la fréquentation d'écoles non juives, l'athéisme, là où son maître, le tzadik de Rimanov, soutenait que la victoire de Napoléon allait apporter la délivrance 3 .

Le magid de Koznitz prit position de manière spectaculaire, au moyen d'un jeu de mots demeuré fameux. À la fête de Pourim, en 1812 (c'était, cette année, le 27 février), quelques mois avant l'arrivée des troupes françaises, lisant le passage du livre d'Esther où la femme et les amis d'Hamman lui prédisent qu'il tombera ${ }^{4}$, il lut en effet, au lieu $\mathrm{du}$ «nafol tipol» du texte, «Napoleon tipol», «Napoléon, tu tomberas $»^{5}$ ! Une autre tradition rapporte que l'épisode advint pendant la lecture de la Torah, le $1^{\text {er }}$ février 1812; le magid aurait dit, au lieu de «Navol tibol ${ }^{6} »$, «Napoleon tipol».

1. C'était le souhait de Rabbi Chlomo de Karlin, le magid de Koznitz Rabbi Yisraël, Rabbi Lévi Yitz'hak de Berditchev. Nous empruntons ici à Yanki Tauber, «Is Judaism a Theocracy?», disponible sur www.chabad.org, et à Nissan Mindel, Rabbi Schneur..., op. cit.; on y lit que «most of the Chasidic leaders in Poland and Austria welcomed Napoleon» (1, p. 251).

2. Voir sur ce point les conclusions de Jean Baumgarten, La Naissance..., op. cit.

3. Voir Jacques Gutwirth, La Renaissance du 'hassidisme. De 1945 à nos jours, Paris, O. Jacob, 2004, p. 20, qui s'appuie sur l'irremplaçable Simon Dubnov, Geschichte des Chassidismus, Berlin, Jüdischer Verlag, 1931.

4. Esther, 6, 13: «Tu ne pourras l'emporter [sur Mardochée]; au contraire, tu t'écrouleras entièrement. »

5. Sur cet épisode fameux, voir par exemple, dans Martin Buber, Gog..., op. cit., le passage sur «Kosnitz et Napoléon», p. 309-316; Henri Kahn, «Les Rabbis...», art. cit., p. 35.

6. À propos de Moïse, dans Exode, 18, 18: «Tu succomberas certainement» (parashat «Yitro»). 
Notons enfin qu'il était courant de voir la guerre contre Napoléon comme une guerre entre Gog et Magog, qui, selon la tradition, annonce la venue du Messie ${ }^{1}$. Pareille approche, très présente par exemple chez Rabbi Na'hman de Bratslav's déplace le problème, par rapport aux questionnements politiques dont il a été ici question jusqu'à présent: la question devient alors celle de savoir si l'on doit ou non hâter la fin de ces guerres (pour hâter l'arrivée tant attendue des temps messianiques). Dans cette optique, il se pourrait bien que le soutien apporté par Mena'hem Mendel de Rimanov à Napoléon tienne à ce que l'empereur apporte la délivrance ultime (geula), désirable fût-ce au prix de nombreuses souffrances.

\section{Partages}

Essayons de lâcher un peu le fil historique pour, à partir de ce cas, réfléchir sur la question générale du rapport à l'émancipation dans le monde juif orthodoxe. L'opposition est nette, dans la vision du «paysage mental» qui y a cours, qu'il s'agisse des 'hassidim ou des mitnagdim, entre, d'une part, le spirituel, la Torah, l'âme, et, d'autre part, le matériel, le confort, les biens. Ce partage n'empêche pas que ce qui est matériel soit élevé - c'est la fonction de l'accomplissement d'un précepte, la mitzva, que d'élever et de sanctifier la matérialité. On lit en outre dans les Pirké Avot que «s'il n'y a pas de farine, il n'y a pas de Torah; s'il n'y a pas de Torah, il n'y a pas de farine ${ }^{3} »$; il ne s'agit donc pas de mépriser la condition matérielle car, méprisable en elle-même, elle est bien l'indispensable accès à la Torah et gagne ainsi sa respectabilité. Le même traité recommande nettement de marier les deux, faute de quoi la Torah perdrait sa valeur ${ }^{4}$. On pense ici à une opposition formulée classiquement, dans la langue de l'étude, comme opposition entre la «voie du monde» et l' «étude», précisément. Cette opposition recoupe, comme sa formulation consacrée, la distinction entre spirituel

1. Ézéquiel, 38-39, et Zacharie 14. Voir Martin Buber, Gog..., op. cit.; et Henri Kahn, «Les Rabbis...», art. cit.

2. Voir Roland Goetschel, «L'hostilité...», art. cit., p. 276.

3. Pirké Avot, 3, 17. Voir le commentaire en ce sens de Rabbénou Yona.

4. Pirké Avot, 2, 2. 
et matériel. Mais soulignons que cette opposition amène une question qui ne se pose en fait pas avec moins d'acuité dans un monde juif - témoin la réflexion qui fut produite sur ce thème dans les textes traditionnels. La question du rapport aux nations et donc de l'assimilation est parfois confondue avec celle du rapport à entretenir avec la «voie du monde», question posée tant avant l'exil que pendant celui-ci; au contraire, l'exil renouvelle la question du rapport aux nations.

Les maîtres enseignent la nécessité de pratiquer les mitzvot de façon continue: pas de partage entre l'activité séculière et une «pratique religieuse» qui occuperait une partie du temps. Même les pécheurs sont pleins de mitzvot comme la grenade de ses grains ${ }^{1}$. Les émancipateurs défendent une tout autre «géographie» de l'existence: on distingue entre divers domaines - le religieux, le politique, l'économique, le familial, etc., toutes sphères que l'on peut isoler, et même, pour certaines, privatiser (la religion et la foi, mais aussi l'intimité, etc.), si bien que, selon ce modèle, l'obtention de l'émancipation, fait qui relève du politique, n'a aucune raison d'entraîner l'assimilation, entendue comme abandon des pratiques juives, qui relèverait, elle, du religieux. L'essentiel est bien là, dans cette divergence de "géographies de l'existence ». Le modèle proposé est tout différent du modèle religieux, qui ne fait pas de place pour une notion du «politique» déprise du fait juif. C'est particulièrement vrai du 'hassidisme, qui insiste sur la dimension messianique du judaïsme: donnant la première importance à la tension entre le présent et l'avenir et à la responsabilité de la génération présente de faire advenir le salut et la délivrance, il ne fait guère de place à la politique, ou du moins construit un messianisme fort divers de celui qui se construit au même moment dans les États-nations². Plus généralement, l'orthodoxie, quelle qu'elle soit, construit un univers mental où il n'est de gain spirituel que dans le domaine de la Torah; et de gain temporel appréciable que débouchant sur un gain spirituel. À cette seule aune sera mesurée l'émancipation, qui n'est, au mieux, qu'un moyen, et qui peut aussi bien, si elle ne satisfait pas à ce critère, être rejetée 3 .

1. 'Hagiga, 27a.

2. Sur ces thèmes, nous devons faute de place renvoyer uniquement à Jean Baumgarten, $L a$ Naissance..., op. cit., et Pierre Bouretz, Témoins du futur. Philosophie et messianisme, Paris, Gallimard, 2003.

3. Ces aspects sont déjà évoqués dans Simon Schwarzfuchs, Napoleon..., op. cit., p. 176: dans l'esprit de Chnéour, «[...] the only important value in life was complete and utter devotion do Judaism and to the fulfilment of its teachings». 
Mais dans les conditions de la modernité, quelle peut-être la réalisation effective de ce modèle traditionnel ? Il fonctionnait aisément quand d'autres, refusant l'émancipation aux Juifs, permettaient de fait sa mise en place; mais après que la situation a changé, ce modèle doit instaurer une tension, en désignant le monde où sont versés les Juifs comme un monde qui n'est pas le leur, en les plaçant dans un ailleurs - ce qui suppose de nier qu'ils se définissent également par rapport à leur cadre de vie réel. Et cet ailleurs, indissociable de l'exil, est considéré comme dangereusement assimilateur. Que l'acquisition de droits civils et politiques conduise à une réduction de la vie juive ne va pourtant pas de soi. Que désigne donc cette incompatibilité ? Les textes sont loin d'être explicites, on l'a vu. Deux idées peuvent toutefois être dégagées: d'une part, l'idée que l'émancipation n'est jamais «offerte» seule, mais accompagnée d'un certain nombre de contraintes, lourdes de risques pour l'identité juive (service militaire, obligations de type éducatif, etc.); d'autre part, sans même parler de ces contraintes, l'idée que, de mille et une manières, cette concession de droits civils et politiques, stimulant le contact avec les non-Juifs, va (veut?) entraîner l'acculturation et l'assimilation. Cela renvoie à des phénomènes situés au niveau des mentalités (avec par exemple la construction de la laïcité: l'élaboration d'un espace «religieusement neutre» et le rejet du fait religieux dans la sphère privée) et des institutions (l'exemple le plus parlant étant sans doute celui de la juridiction, aspect majeur de la souveraineté, dont l'État émancipateur n'admet nul partage); et enfin, point décisif, relevant de réalités sociologiques et économiques, cela renvoie à l'ouverture de possibilités nouvelles pour l'action des Juifs dans la société. La ruse de l'émancipation serait ainsi d'offrir aux Juifs de nouveaux espaces où se perdre:

Ce n'est que lorsque ses individus reportèrent leurs aspirations sociales du contexte de leur propre communauté sur celui du milieu environnant non juif qu'un changement décisif dans l'histoire de la société juive se produisit. Ce fut le cas lorsqu'elle commença à considérer la société non juive non seulement comme un cadre d'activité économique mais également comme une source de gratification sociale ${ }^{1}$.

1. Jacob Katz, De la tradition..., op. cit., p. 364. 
Les choses étant dites ainsi, on voit mieux quelle violence exprime l'émancipation: le parallèle paraît évident avec celle qui est faite aux peuples colonisés. Empruntons aux penseurs du fait (post-)colonial le concept de «double consciousness ${ }^{1} »$. Un phénomène de récupération identique s'est peut-être produit en littérature, où, après une génération de maskilim regardant avec mépris l'âge du shtetl, la «génération de Bialik» a au contraire regretté, dans les deux dernières décennies du $\mathrm{XIX}^{\mathrm{e}}$ siècle, cette époque perdue, et l'a évoquée avec nostalgie et affection, au point qu'on puisse parler d'une «conscience écartelée» de Bialik $^{2}$. Accepter l'émancipation, accepter de «marcher» dans «la voie du monde», c'est affronter, bon gré mal gré, les difficultés de la «conscience double». On connaît la réaction du père de Pierre VidalNaquet, écrivant dans son journal, en 1942, qu' "[il ressent], comme Français, l'injure qui [lui] est faite comme Juif ${ }^{3} »$ : mieux encore que le «Juifs dedans, citoyens dehors» de la néo-orthodoxie ${ }^{4}$, quelle forte expression de cette ambivalence!

L'ambition de la Révolution fut sans doute de faire connaître aux Juifs une évolution radicale: Jews into Frenchmen, dirait-on pour paraphraser un ouvrage fameux ${ }^{5}$. Manière aussi d'affirmer que l'on ne pourrait être l'un et l'autre à la fois 6 .

1. Ce concept forgé par William Edward Burghardt Du Bois qualifie à l'origine la sensation du Noir confronté aux stéréotypes blancs tout en étant, dans une autre perspective, parfaitement semblable aux Blancs. Le fait post-colonial a fait l'objet d'une réflexion dans Labyrinthe (Laurent Dubreuil dir., Faut-il être postcolonial?, 24, 2006).

2. Nous empruntons à une conférence de la Société des études juives prononcée par $\mathbf{M}^{\mathrm{me}}$ Ariane Bendavid à Paris en 2007. Voir aussi, sur le thème de la nostalgie, Richard I. Cohen, « Nostalgia and "return to the ghetto" : a cultural phenomenon in Western and Central Europe», dans Jonathan Frankel et Steven J. Zipperstein dir., Assimilation and Community. The Jews in Nineteenth-Century Europe, Cambridge, New York, Port Chester, Melbourne et Sydney, Cambridge University Press, 1991, p. 130-155.

3. Lucien Vidal-Naquet, «Journal de Lucien Vidal-Naquet (15 septembre 1942-29 février 1944)», éd. Pierre Vidal-Naquet, Réflexions sur le génocide (Les Juifs, la mémoire et le présent, 3), Paris, La Découverte, 1995, p. 111-152, p. 113.

4. On appelle ainsi le mouvement dont la principale figure est le rabbin Samson Raphaël Hirsch (1808-1888), qui entendait marier respect de l'orthodoxie religieuse et intégration dans les sociétés modernes (c'est le sens du slogan «Torah im derekh eretz», «la Torah avec la voie du monde»).

5. Eugen Weber, Peasants into Frenchmen : the Modernization of Rural France, 1870-1914, Stanford, Stanford University Press, 1976, traduction française La Fin des terroirs : la modernisation de la France rurale 1870-1914, Paris, Fayard, 1983.

6. Voir les accusations sur la «double allégeance» et les thèmes du double amour et de la double appartenance. Sur la complexité de la situation juive en exil, voir la source fondamentale que constitue Simon Dubnov, Lettres..., op. cit., en particulier la lettre II, «Le peuple juif en tant que nation spi- 


\section{Jougs et libertés}

Laissons de côté ici les raisons du spectaculaire revirement du mouvement 'habad, qui est devenu la branche 'hassidique la plus moderne qui soit. Du reste, on peut déjà voir dans l'attitude de certains 'hassidim du début du XIX ${ }^{e}$ siècle la preuve de la sécularisation des Juifs, qui, face à la modernité, sont contraints d'entrer en politique: c'est là tout le paradoxe d'une critique du monde moderne qui, pour porter, doit «réinventer» la tradition et entrer dans le monde ${ }^{1}$.

L'orthodoxie propose en somme un travail sur une forme de «servitude volontaire»: refuser l'émancipation serait aimer sa servitude. On retrouve un thème paradoxal très présent dans le discours religieux, celui du «joug des préceptes », du «joug de la royauté des cieux», dont l'acceptation fait bien sûr l'objet d'une forte valorisation, par contraste avec l'usage négatif du mot yiddish «frei», «libre», employé pour désigner celui qui ne se soumet pas à ce joug. Dans le même ordre d'idées, on lit dans les Pirké avot:

Rabbi Né'honia Ben Hakana disait: «Celui qui accepte le joug de la Torah se verra préservé du joug des autorités et des lois naturelles².»

On observe une tension entre la désapprobation qu'encourt celui qui s'affranchit du «joug des préceptes» et l'amour de la liberté (voir la perpétuelle réitération de la sortie d'Égypte: il convient de se libérer chaque jour de la servitude). La solution, dialectique, est que l'asservissement au joug des mitzvot est gage de liberté: selon une célèbre interprétation michnaïque ${ }^{3}$, la loi équivaut à la liberté ${ }^{4}$. A contrario, il

rituelle (historico-culturelle) parmi des nations politiques», p. 117-142; et voir la réflexion intéressante de Phyllis Cohen Albert, «L'intégration et la persistance de l'ethnicité chez les Juifs dans la France moderne», dans Pierre Birnbaum dir., Histoire politique des Juifs en France : entre universalisme et particularisme, Paris, Presses de la Fondation nationale des sciences politiques, 1990, p. 221-243. 1. Nous suivons ici les conclusions de Jean Baumgarten, Récits..., op. cit.

2. Pirké Avot, 3, 5. Rambam assure que Dieu protège du pouvoir des princes celui qui accepte le joug de la Torah, tandis que le Maharal parle d'une possibilité pour l'homme de se délivrer du joug du monde matériel en s'élevant au-dessus de lui.

3. C'est-à-dire qu'on la trouve dans la mishna, traditionnellement définie comme la mise par écrit de la tradition orale de la Torah, donnée par Dieu à Moïse sur le mont Sinaï en même temps que la Torah écrite. 4. Exode, 32,16 ( «Et ces tables étaient l'ouvrage de Dieu; et ces caractères, gravés sur les tables, étaient des caractères divins »), que les Pirké Avot, 6, 2, proposent de lire en remplaçant 'harut, «gravée», par 'herut, «liberté». Cette question est bien le thème central de Emmanuel Levinas, Difficile Liberté, op. cit. 
convient de refuser l'émancipation donnée par l'autre, qui est aussi un asservissement. Refuser l'émancipation que les autres veulent pour soi, c'est aussi s'émanciper.

Retournons à l'histoire. Il y eut, après la destruction du Temple, une longue période sans ghetto, durant laquelle on ne sache pas que le judaïsme ait disparu; une longue période où l'on s'en passa sans qu'il y eût, semble-t-il, d'assimilation massive. Le ghetto ne parait-il pas nécessaire aux Juifs dont le judaïsme est faible et aux chrétiens que le judaïsme angoisse? Un judaïsme affirmé dans un monde sans ghetto fut possible - à Troyes au $\mathrm{XI}^{\mathrm{e}}$ siècle, à Florence au $\mathrm{XV}^{\mathrm{e}}$, par exemple : mais c'était, il est vrai, avant que les Lumières ne réinventent l'universalisme, et en particulier avant que la Révolution française ne propose ce modèle si particulier d'émancipation, dont la réticence à faire place aux particularismes demeure perceptible. 